Portland State University

PDXScholar

Center for Urban Studies Publications and

Reports

Center for Urban Studies

$11-2002$

\title{
Building and Maintaining a Statewide Transportation Framework
}

\author{
Kenneth Dueker \\ Portland State University \\ Paul Bender \\ Portland State University
}

Follow this and additional works at: https://pdxscholar.library.pdx.edu/cus_pubs

Part of the Geographic Information Sciences Commons, Transportation Commons, and the Urban Studies and Planning Commons

Let us know how access to this document benefits you.

\section{Citation Details}

Dueker, Kenneth and Bender, Paul, "Building and Maintaining a Statewide Transportation Framework" (2002). Center for Urban Studies Publications and Reports. 4.

https://pdxscholar.library.pdx.edu/cus_pubs/4

This Working Paper is brought to you for free and open access. It has been accepted for inclusion in Center for Urban Studies Publications and Reports by an authorized administrator of PDXScholar. Please contact us if we can make this document more accessible: pdxscholar@pdx.edu. 


\title{
BUILDING AND MAINTAINING A STATEWIDE TRANSPORTATION FRAMEWORK
}

\author{
Kenneth J. Dueker \\ Paul Bender \\ Portland State University
}

November 2002

\section{Submitted for Presentation at the 2003 Annual Meeting of the Transportation Research Board}

\author{
Center for Urban Studies \\ Portland State University \\ Portland OR 97207-0751 \\ http://www.upa.pdx.edu/CUS/
}

The Washington State Department of Transportation and United States Geological Survey(USGS) provided support for this research. The views and conclusions contained in this document are those of the authors and should not be interpreted as representing the opinions or policies of the U.S. Government or the State of Washington. Mention of trade names or commercial products does not constitute their endorsement by the U. S. Government or the State of Washington. 


\begin{abstract}
Creating and maintaining up-to-date sharable Geographic Information SystemsTransportation (GIS-T) data is challenging. Many states are working on Transportation Framework efforts to build a complete, consistent, and current transportation data layer in conjunction with the NSDI and Geospatial One-Stop efforts. This paper summarizes an effort that systematically re-examined transportation data sharing issues as part of the development of a Transportation Framework for the State of Washington. Business needs were assessed in terms of spatial and temporal accuracy needs of stakeholders and users of a state-wide Transportation Framework. A conceptual model was developed for a Transportation Framework with emphasis on data flows to update and maintain it and other transportation databases. The paper concludes with a synthesis of issues, including determining the desired robustness of the Transportation Framework and cost allocation principles for possible add-on enhancements.
\end{abstract}




\section{Introduction and Purpose}

This paper assesses and summarizes alternative approaches and data sources for the development of a Transportation Framework for the State of Washington (1). The original purpose of this assessment was to systematically re-examine transportation data sharing issues that had been discussed among Oregon and Washington State agency stakeholders at length, and in a manner to foster decision making. The resulting white paper found that in some cases, choices among alternatives may require more detailed analysis or pilot studies. The development of both this summary and the full white paper benefited from discussion at the Interorganizational Resource Information Coordinating Council (IRICC) Roads Committee, the road database coordinating body for Washington and Oregon salmon enhancement planning efforts as well as other bi-state road data needs.

\section{The Scope and Rationale of a Statewide Transportation Framework}

It is anticipated that a statewide Transportation Framework will have two purposes. First, the Framework can be considered one of a set of National Spatial Data Infrastructure (NSDI) coordinated map layers comprising point, line, and area objects representing the location and extent of transportation features that is single, complete, consistent, and current. Single means a common definition of features in the Transportation Framework and a core set of attributes about the features. Consistent means a known level of spatial and temporal accuracy with proven updating mechanisms. The result is consistency in spatial representation and temporal currency. 
The primary function of a common Transportation Framework is to provide a source of "best available" linework and attribute data that would be updated periodically, probably on an annual basis. This will support a core set of transportation applications that are common to a sufficient number of organizations to warrant a common database.

This Transportation Framework representation would serve three types of business needs: planning, operations, and management. Planning type business needs will be met for a limited range of transportation and non-transportation organization stakeholder applications. The representation could also support a limited number of operations type applications, such as pathfinding for rerouting and permitting. Individual users can assess it for fitness to their application. In many instances the Transportation Framework may need augmentation for specific applications. For example, many business needs, such as transportation planning, congestion management, etc., require at least a bi-directional centerline if not dual carriageways or even individual lanes, either in the basic geometry or by attribution. These needs may be too specific or time sensitive to include within general use data for which the Framework is responsible.

The second purpose of the Transportation Framework is to facilitate updates to the Transportation Framework itself, and to other more application-specific transportation databases. This entails a Clearinghouse of new or modified road features that is collected in the form of updating transactions to the database. These transactions would be derived from construction projects undertaken by or on behalf of transportation organizations. They are then accumulated in the Clearinghouse, and used to update the Transportation Framework's representation of the transportation system. In addition, local, state and federal organizations and road database 
vendors that maintain their own transportation databases could select updates for transportation features deposited by all transportation organizations for their region of interest.

This twofold approach is needed to facilitate updating the best available linework, while at the same time making more detailed data pertaining to new and modified transportation features available. This will support those who need updates of more detailed content and greater spatial and temporal accuracy. The challenge is to create incentives and/or mandates to report new and modified transportation feature data to the Clearinghouse on a transactional basis. In part this can be done by providing guidance on the proper form of formatting and reporting of these changes.

\section{State Framework Review}

This section reviews other efforts at creating state Transportation Frameworks, some of which are also aimed at adopting and/or testing the Federal Geographic Data Committee (FGDC) transportation identification standard (2). Two approaches are noted. The first represents state Departments of Transportation (DOTs) that build statewide Geographic Information Systems (GIS) databases of all roads for internal reasons. These can be seen as indirect attempts to create a statewide Transportation Framework. The second approach is to build a comprehensive Transportation Layer within the context of a statewide FGDC-inspired national Spatial Data Infrastructure (NSDI) Framework and Geospatial One-Stop efforts. Both of these efforts are reviewed here.

Several states have embarked on developing statewide GIS databases of all roads.

Dueker, Butler, Bender, and Zhang (3) summarize some key points from three states: Minnesota, Wisconsin, and Arizona. These states are leading the way because of their early start in tackling 
the work. They are starting from existing mainframe highway inventory and mapping applications, while enhancing and converting to a GIS application. Several other efforts are described briefly below.

Texas is developing the Texas Strategic Mapping Program (StratMap) to compile what it terms "mission-critical" GIS Framework data, including transportation, for the entire state. An integral part of the StratMap objective is the "open exchange of information between agencies, open access to non-sensitive government information, and private sector value-added opportunities."

Both Vermont and Montana have made significant progress in testing Framework implementation using FGDC schema for identification. Vermont has recently completed its pilot project. Montana began a similar FGDC Framework review pilot titled "A Collaborative Multijurisdictional Approach to Building a Geospatial Ground Transportation Framework Database for Montana.’(4).

Kentucky has recently adopted an enterprise architecture perspective and has developed its own spatial data standard, an integrated model of multi-thematic data content standards. The spatial data standard represents an implementation of the Federal Geographic Data Committee geospatial data content standards and meets data sharing requirements of the NSDI. Beyond this, Kentucky DOT is creating a complete street Centerline file using GPS.

Utah has made an effort to develop the Utah Framework Implementation Plan, based on the seven FGDC NSDI Framework layers. The transportation Framework effort is adhering to the FGDC standards and data model. Utah is also involved in a transportation pilot study testing the USGS National Map. The only other transportation pilot study is the Washington-Idaho 
National Map pilot, which includes Spokane and Pend Oreille counties in Washington, and Kootenai and Bonner counties in Idaho.

The Washington-Idaho National Map pilot will explicitly attempt to build up the Framework map from as many local sources (city, county, state and Forest Service) as possible. Any gaps in available data will be filled in with data purchased from a vendor, Geographic Data Technology (GDT). Data sharing partnership agreements and incentives to participate are seen as key organizational elements to be tested. An ArcIMS NSDI clearinghouse node is being considered as the data server for the pilot.

In summary, these state efforts provide guidance on the initial building of a comprehensive and complete statewide transportation layer. They are very expensive to build, but in spite of this, little attention as of yet has been given to complex and costly issues of updating. The second approach, that of attempting to test compiling data from numerous sources following the FGDC Road Identification schema, is moving ahead more slowly, as state Geographic Information Councils work to tackle both technical and organizational issues that so far have hampered Framework construction. From this review we cannot yet deem either approach as being successful in meeting the scope of the Transportation Framework, particularly the updating requirements and the business needs of potential clients.

\section{Business Needs}

Business needs of users of transportation data were examined to determine the content, structure, and spatial and attribute accuracy requirements for the Transportation Framework. The challenge is to determine how many and what needs to accommodate in a single 
representation of the transportation system. Building a robust multi-purpose representation satisfying all users would be costly and difficult and would demand frequent updates. On the other hand, too basic a representation might not serve enough needs to be justifiable.

Transportation databases are built to support of some combination of planning, management, and operations needs. Generally, the business needs of planning can usually be met with spatial data of low or medium spatial and temporal accuracy. Another generalization is that the business needs of non-transportation organizations require less accurate spatial and temporal transportation data than do transportation organizations (agencies that own and maintain roads, such as departments of transportation, public works and U.S. Forest service and timber companies), with the major exception of emergency dispatch (E 911). These conclusions follow from an assessment of the business needs and applications discussed below.

Although the business needs supported by a Transportation Framework could be limited to requirements that are inter-organizational in nature, intra-organizational data sharing may be a stronger incentive to motivate organizations to participate. Many organizations have internal stovepipe GIS that could benefit from better sharing of data. Improving data sharing within the organization would thereby foster inter-organizational data sharing capacity.

A preliminary examination of business needs within Washington State Department of Transportation (WSDOT) exemplifies opportunities for data sharing within and outside of the Transportation Framework. The business needs of WSDOT fall in the following categories:

- The need to relate state roadway data to other layers, such as land ownership, local roads, wetlands, streams, land use and land cover, utilities, and sensitive environmental and cultural areas.

- The need for a detailed inventory of infrastructure on state roads. 
- The need for a complete GIS representation of all roads in the state in a form to support routing that includes functional and jurisdictional classification, surface type, status, and height, weight, and turn restrictions.

Meeting these internal business needs requires sharing of data within WSDOT and with others. Achieving the internal data sharing will make the external data sharing easier and more effective. WSDOT will need to address which business needs can be derived from Framework data, and which will need more detailed content or more frequent updates than can be provided by the Framework, and thereby maintained internally, outside of the Framework.

WSDOT should be able to take advantage of the Framework in satisfying its business needs. They need to relate transportation layers to other Framework layers. They need a comprehensive GIS-based infrastructure inventory system as well as a complete, consistent, and current representation of roads suitable for routing applications. A more detailed assessment of business needs for transportation has been conducted by WSDOT. This included extensive involvement of state and local stakeholders (5).

The requirements of several statewide or regional applications for transportation data were examined to identify common transportation data elements and spatial and temporal requirements to include in the Transportation Framework. These applications are emergency management, infrastructure management, freight mobility, and salmon enhancement. All four are illustrative of the growing and diverse applications of transportation data. Emergency Management and Infrastructure Management are the most common GIS-T needs across the country, and so are discussed in some detail below, along with the new Homeland Security initiative. 


\section{Emergency Management Business Needs}

Emergency Management requires transportation data for two distinct business needs: disaster planning and emergency response. Disaster planning is an important form of contingency planning that deals with evacuation routing and rerouting around closed facilities. Contingency planning does not require the same level of spatial and temporal accuracy as emergency response. Emergency response has a higher need for current data and has a higher need for spatial accuracy to snap GPS-derived positions to the correct piece of road. For emergency response, temporal accuracy is the highest requirement. The most recent streets and corresponding addresses are required for accurate address geocoding of emergency calls and routing. The spatial accuracy requirement for disaster planning is to assess road and bridge impacts from floods, fires, and earthquakes. The temporal accuracy requirements for contingency planning are not real time.

Disaster planning makes increasing use of GIS as a means of quickly integrating and sharing data among agencies. Road centerline files help determine evacuation routes and answer spatial questions or queries, such as: What roads are subject to flooding? Which routes are already designated emergency routes (for plowing, etc)? What are evacuation times of main/alternative routes? Where are bridges (possibly impassable) located? What roads are affected by disaster? Are they totally impassable? What alternative routes are available?

This distinction between business needs of planning and operations is crucial. For example, disaster planning is a planning business need of emergency management, while emergency response is an operational business need, and there are distinct differences in their spatial and temporal accuracy requirements. A common Transportation Framework would serve 
the needs of disaster planning, but the needs of emergency response would require more currency, or temporal accuracy than can be supported by the Transportation Framework.

Homeland Security has become a major issue in the arena of emergency management. Homeland Security encompasses both disaster planning and emergency response in the event of a disaster or emergency. The emergency response component of Homeland Security would in all likelihood require a specialized database, to handle specific operational needs of Homeland Security. The representation in a state or national Transportation Framework accessed through the Geospatial One-Stop portal (6) used for contingency planning would be a good starting point.

\section{Infrastructure Management Business Needs}

The business needs of infrastructure management are diverse and complex. Infrastructure management is a concept that pertains to organizations responsible for planning, construction, maintenance, and operation of infrastructure, such as departments of transportation and public works. They tend to require significant levels of inter-organizational coordination, and are thereby candidates for data sharing via the Transportation Framework.

The lifecycle management concept used in infrastructure management consists of the functions of planning, construction, maintenance, and operations. These categories are useful in assessing spatial and temporal accuracy requirements. These can be used to address many applications that fall under the heading of infrastructure management. A new business need of infrastructure management is the Government Accounting Standards Board Statement (GASB) 34 reporting requirements for asset management below.

The recently released asset management GASB 34 requirements reiterate and reinforce the business needs requirements of asset data management. A good working definition comes 
from the FHWA Asset Management primer: "Asset management is a systematic process of maintaining, upgrading, and operating physical assets cost-effectively. It combines engineering principles with sound business practices and economic theory, and it provides tools to facilitate a more organized, logical approach to decision-making” (7). Properly designed and implemented asset management systems can bridge the stovepipe problem of current individual bridge

management systems (BMS) and pavement management systems (PMS) that inhibit the sharing of data that the Transportation Framework, and this paper, are attempting to help address.

Underlying the business needs of Asset Management is the "economic assessment of trade-offs between alternative improvements and investment strategies from the network-or system-level perspective" (7). Some of the basic business needs identified by the FHWA include: inventory of assets (physical and human resources); valuation of assets; quantitative condition and performance measures; and performance-prediction capabilities. An effective Asset Management system, making use of the Framework, has the potential to strengthen the now-tenuous link between the transportation plan and actual programming and resource allocation decisions.

\section{Spatial and Temporal Accuracy}

The Transportation Framework must be consistent in spatial and temporal accuracy with other statewide Framework data and FGDC layers (Administrative Boundaries, Hydrography, Cadastral, Ortho imagery, Elevation, and Geodetic Control). Table 1 provides a first approximation of emergency management application accuracy requirements for the Transportation Framework that takes into consideration consistency with other Framework layers 
Spatial Accuracy needs will vary, according to business needs. Although it is desirable to find the least amount of accuracy necessary to the Framework, a flexible model that accepts (and maintains metadata for) data of varying accuracy is desired.

Temporal accuracy in the context of the Transportation Framework deals with the frequency and method of update. Operational business needs have much more demanding currency requirements. Table 2 illustrates low, medium and high accuracy levels. Generally, a low level of accuracy would meet business needs for planning while a high level of accuracy is needed for operations business needs. Differing requirements in urban and rural areas are also recognized in Table 2.

\section{Conclusions From the Assessment of Business Needs}

The business needs of GIS applications in the areas of natural resource management, infrastructure management, emergency management, and services management applications were assessed in the white paper. The Transportation Framework should focus on supporting planning functions initially, with very limited support for the needs of operations. In a longer timeframe, a more robust Transportation Framework, one having greater spatial and temporal accuracy and more detailed attribution, could support more management and operations functions. But initially the requirements and standards for the Transportation Framework are translated to spatial data set and GIS functional requirements that support planning functions. These requirements are both a consistent spatial and temporal accuracy across Framework layers, and a consistent representation of transportation across organizations. The initial requirement for a Transportation Framework is to relate to other Framework layers and to other organizations. 
The requirement of consistency with other statewide Framework layers (Administrative Boundaries, Hydrography, Cadastral, Ortho imagery, Elevation, and Geodetic Control) includes:

- Administrative boundaries that fall on streets should align with the Transportation Framework's representation of those streets.

- Hydrography and Transportation should relate correctly, i.e. the stream on the correct side of the road and the steam crossings at the correct river and road mile points.

- Centerline representations of transportation features should fall within rights-of-way of Cadastral layers.

- Centerline representations of transportation features should relate correctly to Ortho imagery.

- The elevation attributes of transportation features should be consistent with the Elevation layer and topographic maps generated from it.

- The temporal currency of transportation features should be as or more current than the other FGDC layers.

- The Transportation Framework should support routing applications for contingency planning.

The requirement of a consistent representation of the Transportation Layer across organizations requires that organizations agree on fundamental elements of transportation in order to exchange data. This consists of the following:

- Criteria for segmenting and identifying roads, i.e. the need to define a transportation feature to facilitate inclusion, identification and exchange of data. 
- Consensus on treating transportation features and their intersections as logical objects that can be represented at larger scales as divided roadways with details of ramps and lanes.

- Consensus on some minimum level of network topology and link and node attribution of restrictions for simple routing.

- Consensus on the frequency of updating the Transportation Framework.

- Consensus on methods of identifying additions, changes, and deletions of transportation features and sharing updates.

- Consensus on the linear referencing methods to locate attributes along transportation features.

- Consensus on selected attributes of transportation features that are needed by most organizations.

It is important that all Framework participants acknowledge that the Transportation Framework is not intended to be a replacement for their transportation databases, so it does not have to, nor should it, contain the detail or the robustness to satisfy all their applications. Yet their databases may be derived from the Transportation Framework and should be updated from transactions from the Clearinghouse of new or modified transportation features.

Those organizations that contribute data to the Transportation Framework are assured that other organizations have access to the most current and accurate inter-organizational data. Those organizations that access data from the Transportation Framework are likewise assured that they have access to the most current and accurate inter-organizationally planning data that is available. Similarly, within organizations, there is a need to share data to avoid the problems of 
stovepipe systems that duplicate basic data and lead to inconsistent representations with varying degrees of spatial and temporal accuracy.

We are unable to conclude which is the preferred method of compiling the complete, consistent and current representation of the Transportation Framework (Roads) ${ }^{1}$. Three pilot studies are proposed to assess the different methods of compiling the Transportation Framework (Roads). One way is to have a single contractor or agency compile it. Another way is have each transportation organization add and fit their data. The third way is to hire a road database vendor to abstract or enhance their product to meet Transportation Framework requirements, and to maintain it. Regardless of which approach is chosen (commercial or primary sources) the road vector data will need to be displayed on digital orthophoto imagery for validation.

\section{Rationale For Transportation Framework (Roads)}

The process for deciding on the structure, content, and detail of the Transportation Framework has been ongoing and challenging, with constant tension between keeping the Framework basic or making it more robust. The argument for simplicity is driven by natural resource applications that merely need "best available" linework for roads to serve as reference data. Yet, when the business needs of these organizations are examined more closely, they often need more robust, intelligent road data to handle routing questions, road ownership or responsibility, surface type, status (planned, under construction, open/closed, retired), bridge/culvert structures, etc. Incorporating these attributes into the roads database increases the importance of updating the data. Consequently, it is difficult to keep the Framework basic.

\footnotetext{
${ }^{1}$ The remainder of the paper addresses roads only.
} 
Meeting the more demanding business needs of transportation organizations requires even more data. The routing of overweight/oversize vehicles requires weight/height restrictions, and road maintenance requires a detailed inventory of roadway infrastructure.

This tension between simplicity and robustness resulted in the realization that a complete, consistent and current representation of roads is needed, but the Transportation Framework cannot meet all business needs for road data. Yet, the Framework should play a major role in collecting data concerning new or modified roads that will enable updating and improving the complete representation of roads. This led to the notion of a clearinghouse for data on new and modified roads (3) that would serve to update and improve the "best available" data on roads, and to serve as a resource to others who maintain a more detailed roads database.

This learning process led to the establishment of the following criteria and design principles for the Transportation Framework (Roads):

- Compile "best available" data from existing imagery and GIS resources to create a complete, consistent, and current roadway system. Attribute it minimally to support simple routing and address geocoding applications.

- Enable its gradual improvement in spatial accuracy and correspondence with other layers on an as-needed, ad-hoc basis by means of a check-out/check-in process for regions undergoing detailed study.

- Enable the addition of content detail and spatial accuracy from engineering ComputerAided Drafting and Design (CADD) data and inventory databases. This requires that the Transportation Framework includes a roadway identification schema and linear referencing. 
- Establish an explicit periodic versioning process to update the complete representation of the road system.

- Capture data when roads are created or modified in the form of transactions Begin the capture of these data on a day-forward basis.

- Create a clearinghouse of these transactions from which other road database users can query and select data to maintain and update their own organizations' data.

- Create incentives, mandates, guidance, and technical assistance to transportation organizations to foster the reporting of metadata relating to all of the activities outlined above.

\section{The Proposed Transportation Framework Concept}

Figure 1 is a conceptual model of the Transportation Framework (Roads) and its inputs and outputs. There are two major components of the Transportation Framework (Roads). The first, labeled A, is a complete, consistent, and current representation of Roads, and the second, labeled $\mathrm{B}$, is a Clearinghouse of new roads or changes to roads. The diagram illustrates the compiling or building from GIS source material to create the initial Roads database. After this initial build process, the database would be updated periodically from the data collected in the interim by the Clearinghouse of new transportation features.

In addition, there would be checkout procedures for more extensive and complete upgrading for selected regions as warranted. This complete representation of the Road system would be of use for GIS analysis by organizations who wish to use road data, but who do not want to maintain a roads database. On the other hand, there are organizations that need to maintain one or more roads databases for their region of interest, but who find it difficult to 
obtain current data from other organizations that are responsible for maintaining roads within the same region. After implementation of this Transportation Framework concept, these organizations would query the Clearinghouse for jurisdictions within their region of interest, for Transportation Feature types of interest, and for a time period of interest. This more direct way of obtaining data on roads that are new or have undergone change would increase efficiency and effectiveness.

Figure 1 illustrates an evolutionary process that starts with a project $(\mathrm{PJ})$ that utilizes the Transportation Framework (A) and then evolves to a separate roads database to support an ongoing operational program $(\mathrm{PG})$ whose database needs more frequent updating. This program roads database then draws updates from the Clearinghouse (B). Alternatively, an improved project database could be used in a check-out/check-in process to upgrade the Transportation Framework (Roads). The arrow from PJ to the Check-out/Check-in Upgrade box represents this in Figure 1. The diagram shows how transportation organizations input to the Clearinghouse data about new or modified roads for which they are responsible. At the same time they are users of the Clearinghouse for data about new or modified roads that are maintained by others within their region of interest. This concept of a Clearinghouse will enable migration from the standard data versioning update process to a transactional update approach. Figure 2 illustrates this exchange of data among transportation organizations more clearly.

Figure 2 illustrates the user community for the Transportation Framework (Roads) by means of an example for King County, WA. Some of the organizations within King County that own and maintain roads are listed. Under the Transportation Framework concept they would provide data to the Clearinghouse and to other parts of their own organization on roads they have built or changed by means of projects, work orders, or permits. In addition, they withdraw data 
from the Clearinghouse for roads within their area of interest that have been built or changed by actions of other road organizations. Similarly, there are organizations such as Puget Sound Regional Council (PSRC), and private road database vendors, such as GDT and Thomas Bros., who maintain road databases, but who do not maintain roads. Also, there are organizations who maintain neither roads nor road databases, but who need a roads database for GIS analyses. IRICC falls into this category. Emergency dispatch organizations (E911) are a special case. If road updates from the Clearinghouse are timely enough to meet their needs they could be a potential user of the clearinghouse. If not, E911 organizations might be a contributor of data for new roads, particularly if the reporting of road data from transportation organizations is not well recorded or reported.

Not all Transportation Framework (Roads) implementation issues can be fully anticipated. Remaining issues need to be explored in more detail. Six Pilot studies have been proposed to both Washington and Oregon Transportation Framework committees seeking to address these concerns.

The six pilot studies are intended to test several key and interrelated concepts and procedures in building and maintaining the Transportation Framework (Roads). The studies are meant to address issues that have been discussed and in the Washington/Oregon Transportation Framework arena but which need further study. More than one study is proposed to test a single concept or procedure ( e.g., Pilots 1,2,3 test the compilation step), and one will test more than one procedure (e.g., Pilot Study 4 is used to test common format and transaction updating).

These pilot studies will provide empirical and qualitative evidence of what works and what does not in a variety of situations. Cost data and public domain issues that arise will be especially valuable, as these relate to two of the primary objectives of the Framework effort. 


\section{Options and Directions}

Several Transportation Framework constituencies, each with slightly different priorities or business needs, have been identified. How many of these business needs will be supported by the initial Transportation Framework (Roads)? The answer, in part, depends on: 1) willingness to provide funding, staff or database resources, needed to add functionality to meet specific business needs, and 2) the extent to which those business needs are common to several stakeholders. For example, address geocoding is a business need common to several stakeholders, but unnecessary to others.

In addition, these choices of options enable the development of a phased approach to building the Framework, based on identifying and ranking business need priorities. WSDOT has undertaken further analysis of business needs that will assist to distill these priorities and basic needs for the Framework.

Functional "add-on" enhancements to the "best available" roads linework of a Framework will satisfy many GIS needs, including tight integration of the Transportation Framework (Roads) with other NSDI layers. There is great interest and need for integrating hydrography, cadastral, roads, railroads and bridge structures, including culverts, for salmon enhancement planning. In addition, this will include spatial accuracy improvements to the best available linework to support limited vehicle-tracking applications. Three optional enhancements to the basic linework follow directly from the analysis and identification of business needs:

- Address ranges and street names. Address geocoding functionality is of great interest and importance to emergency dispatch agencies and to many other users. 
- Linear Referencing Systems (LRS) to support adding attributes of roads for and infrastructure (IMS) management.

- Network representations of the roadway system to support routing applications, such as disaster and contingency planning. Overweight/oversize truck routing would require additional data of height, weight and turn restrictions.

The three enhancements listed above can be prioritized for phased implementation and to identify stakeholders willing to pay for the enhancement. A rough estimate of cost for compiling the basic linework statewide is estimated to be one million dollars per state (Washington and Oregon). This estimate does not include administration or management of the compilation process. Nor does it include the time and cost of determining exactly what data should be used, setting up data sharing partnerships, and other aspects of incorporating the concerns or stakeholders. Each enhancement is estimated to cost $\$ 250,000$ per state. The additional cost of enhancements should be the responsibility of stakeholders who would benefit.

Regardless of which combination of the three enhancements is selected, instituting an update and maintenance process, such as the transaction update approach for new and modified transportation features is crucial to ongoing maintenance of the Framework. However, the cost estimates for the basic best available linework and the above listed enhancements do not include maintenance costs associated with building and operating the Clearinghouse of new and modified transportation features.

The priority of business needs drives not only the choice among options for functionality, it drives the way in which the Transportation Framework is built, structured, and maintained. 
The following scenarios illustrate the inter-relatedness of business needs, functional options, compilation method, data model, and maintenance method:

- If emergency dispatch is the highest priority, street addressing and relating wireless phone positions to the nearest street becomes the most important functionality of the Transportation Framework. This suggests starting with an existing integrated database, such as GDT or TIGER.

- Giving salmon enhancement planning the highest priority requires a process of stitching together the best available linework with route identifiers and linear referencing to facilitate accessing bridge and culvert attributes from infrastructure management organizations (e.g. city, county, and state DOTs, FS, BLM).

- Ranking both needs equally may lead to consideration of two separate frameworks. These individual frameworks would serve to better handle contrasting and competing needs, balancing desired redundancy and unnecessary duplication.

These scenarios serve to illustrate that the possible choice set is large. The options are not mutually exclusive. Stakeholders will have to mix and match among options and combinations to decide how to best accommodate their business needs to take advantage of a cooperative effort to share costs while at the same time minimizing the loss of control associated with a multi-participant effort. In other words, will the increases in spatial and temporal accuracy of the proposed Transportation Framework outweigh the risks of a multi-participant effort? As this discussion implies, uncertainty and risk inhibit buy in of Framework stakeholders. Consequently, reducing uncertainty and risk is a primary challenge. Meeting this challenge with the goal of achieving stakeholder confidence and support will require agreement on: 
- A clear articulation of stakeholder business needs and the corresponding Transportation Framework functionality.

- Feasible and achievable cost, time, and overall resource estimates.

There are a number of institutional and technical barriers to achieving this consensus. Surmounting them can be difficult. These institutional and technical barriers to address are:

- Integration and conflation of transportation data from different sources and systems with different operational definitions of what a road is, different segmentation criteria, and different spatial and temporal accuracy.

- The need for Framework data to interface with specialized applications with proprietary formats (e.g., infrastructure management, address geocoding, and routing systems)

- Building consensus as to the content of a common framework layer in a multi-participant setting.

- Ever-changing and evolving conditions, expectations, and needs of Framework stakeholders.

- Resource and funding requirements and uncertainties in relation to control and time issues of managing a multi-participant effort.

\section{Conclusions}

This paper summarizes a white paper prepared for WSDOT and provides a synthesis of issues and alternatives in the development of a Transportation Framework. The recommended two-part approach to the Transportation Framework will accommodate pressing applications, 
such as the need for a roads spatial data set for salmon enhancement planning and Homeland Security. At the same time, the Clearinghouse concept for collecting more detailed data on new or modified roads will enable gradual upgrading to a more robust Transportation Framework. In addition, the more detailed data on new roads will provide others with resources to update their own Roads database.

The white paper served the Transportation Framework initiatives in both Washington and Oregon in support of a broad range of applications in resource management, emergency management, infrastructure management, and services management. The white paper defined the purposes of pilot projects needed to test some of the assumptions and issues that are still outstanding. The completion of these pilot studies will help to determine if the proposed twopart approach to the Transportation Framework is workable and feasible.

The conceptual model of a Transportation Framework focuses attention on the importance of updating. Currently, most of the attention has been focused on building rather than updating and maintaining the Transportation Framework. Many state DOTs allocate gas tax revenues to local governments in the form of state aid to local roads. The administration of these programs could mandate submission of data concerning changes to the local road system. Changes occur in three ways: large projects that are part of capital improvement program, smaller projects done internally as work orders, and projects that are done by others through a permitting process, such as dedication and construction of streets as part of a subdivision. Data capture for each project would produce one or more line or intersection features to populate a geodatabase in the Clearinghouse of new or modified roads. 


\section{References}

1. Dueker, K.J., and P. Bender. (2002). "White Paper on Issues and Strategies for Building a State Transportation Framework.” Research Project Report No. 122. Accessed Aug. 1, 2002. http://www.wsdot.wa.gov/gis/transframework/TFwpFINALApril.pdf.

2. Federal Geographic Database Committee. (2000). NSDI Framework Transportation Identification Standard (Public Review Draft). Accessed 3/02. http://www.bts.gov/gis/fgdc/web_intr.html.

3. Dueker, K., Butler, A., Bender, P., \& J. Zhang. (2001). "A Clearinghouse Approach to Sharing Transportation GIS Data," Transportation Research Record, 1768, 203-209.

4. Montana Transportation Working Group. (2002). "A Collaborative Multi-jurisdictional Approach to Building a Geospatial Ground Transportation Framework Database for Montana" Accessed 2/02.

http://mtgeo.org/itwg/framework/Transportation/Grant/app04182000.htm.

5. Washington State Department of Transportation. (2002). "WA-Trans Business Needs Document." Accessed 11/02.

http://www.wsdot.wa.gov/mapsdata/transframework/Business\%20Needs\%20Specs\%20Outli ne0902.pdf.

6. Office of Management and Budget. (2002). Geospatial One-Stop Initiative Website. Accessed 11/02. http://www.geo-one-stop.gov.

7. Federal Highway Administration. (1999). "Asset Management Primer." Accessed 11/02. http://www.fhwa.dot.gov/infrastructure/asstmgmt/amprimer.pdf. 


\section{List of Tables and Figures}

Table 1. Framework Business Needs Sample Table

Table 2. Accuracy Requirements

Figure 1. Building and Maintaining the Transportation Framework (Roads)

Figure 2. Transportation Organizations Contribute and Withdraw Data from the Transportation Framework (Roads) 
Table 1. Framework Business Needs Sample Table

Emergency Management

\begin{tabular}{|l|l|l|l|}
\hline & Planning & Management & Operations \\
\hline Business Need & Disaster Planning & $\begin{array}{l}\text { Response Coordination } \\
\text { \& Reporting (C \& R) }\end{array}$ & Emergency Dispatch \\
\hline Spatial Accuracy & Medium & Low & Med/High House/Highway \\
\hline Temporal Accuracy & Low & Low & High \\
\hline Data Model & $\begin{array}{l}\text { Boundary, } \\
\text { Bi-Directional Flow } \\
\text { Network }\end{array}$ & Thematic Map & $\begin{array}{l}\text { Bi-Directional Flow } \\
\text { Network }\end{array}$ \\
\hline Attributes & $\begin{array}{l}\text { Bridge Height \& Weight } \\
\text { Restrictions }\end{array}$ & $\begin{array}{l}\text { Functional and Jurisdiction } \\
\text { Classes }\end{array}$ & Street Address Ranges \\
\hline
\end{tabular}

Table 2. Accuracy Requirements

\begin{tabular}{|l|c|c|c|c|c|c|}
\hline Type of Region & \multicolumn{3}{|c|}{ Metropolitan } & \multicolumn{3}{c|}{ Non-Metropolitan } \\
\hline Accuracy Level & High & Medium & Low & High & Medium & Low \\
\hline Source Scale & $1: 1000$ & $1: 10,000$ & $1: 24,000$ & $1: 5000$ & $1: 24,000$ & $1: 100,000$ \\
\hline Positional Accuracy (ft) & $1-5^{\prime}$ & $20^{\prime}$ & $40^{\prime}$ & $10^{\prime}$ & $40^{\prime}$ & $100^{\prime}$ \\
\hline $\begin{array}{l}\text { Temporal Accuracy } \\
\text { (update frequency) }\end{array}$ & $\begin{array}{c}\text { less than 1 } \\
\text { minute }\end{array}$ & $1-7$ days & 3 months & $\begin{array}{c}1-5 \\
\text { minutes }\end{array}$ & $2-14$ days & 12 months \\
\hline Linear Accuracy (ft) & $1^{\prime}$ & $5-10^{\prime}$ & $50^{\prime}$ & $5-10^{\prime}$ & $50^{\prime}$ & $250^{\prime}$ \\
\hline $\begin{array}{l}\text { Attribute Detail } \\
\text { (\# of attributes per } \\
\text { segment) }\end{array}$ & $100+$ & $10-100$ & $1-10$ & $100+$ & $10-100$ & $1-10$ \\
\hline
\end{tabular}


Figure 1. Building and Maintaining the Transportation Framework

(Roads)

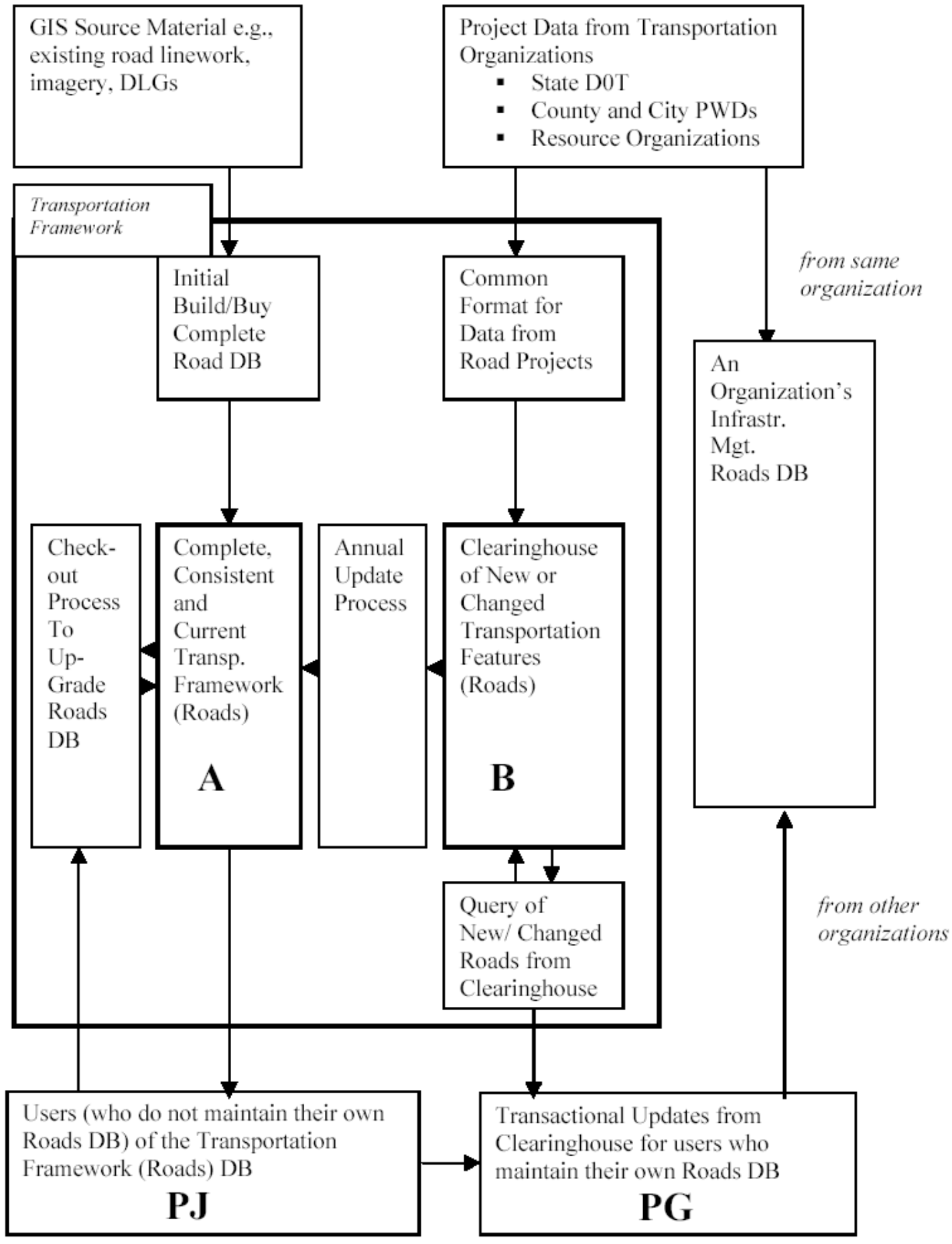


Figure 2. Transportation Organizations Contribute and Withdraw Data from the Transportation Framework (Roads)

1.

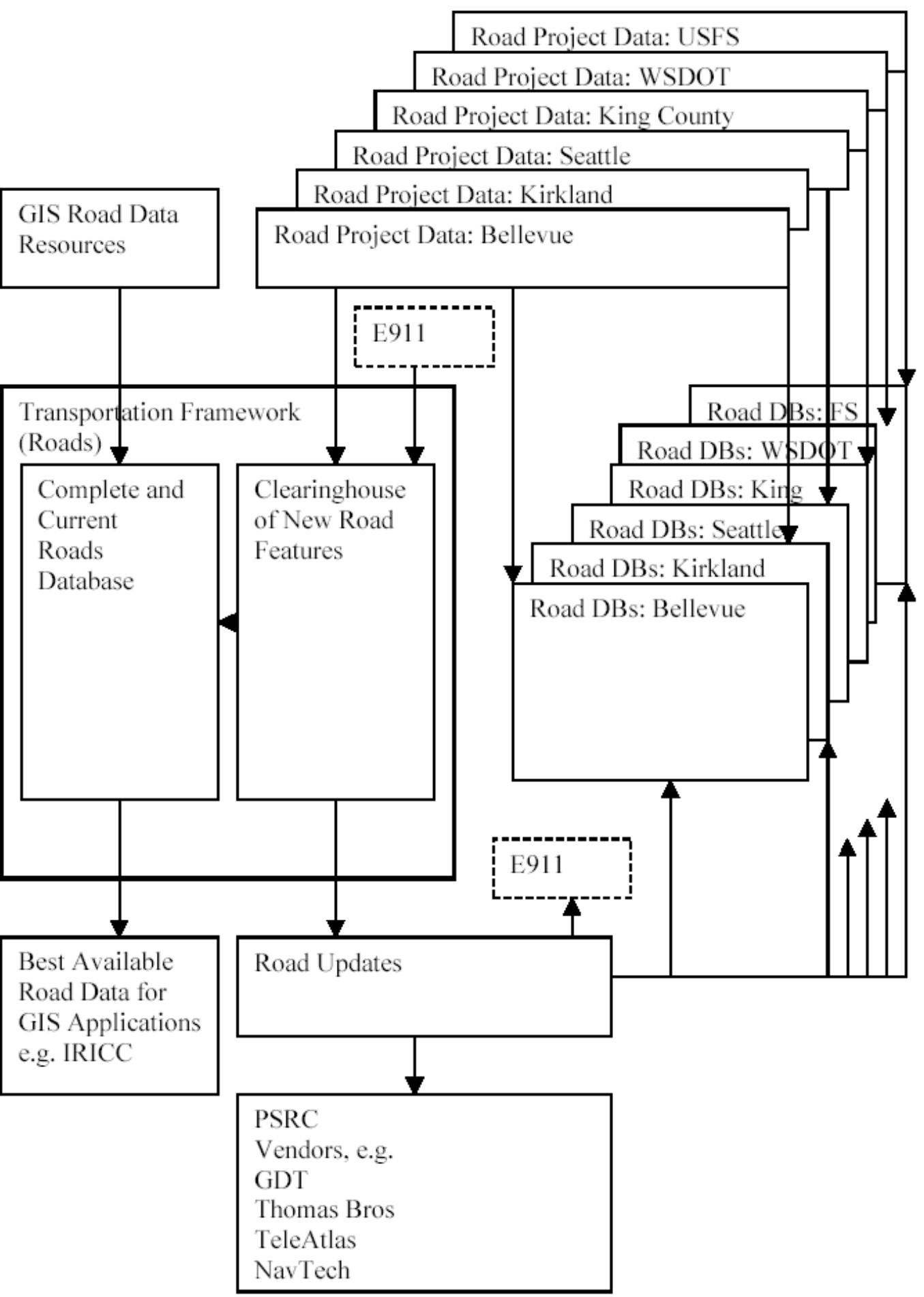

